\title{
Effects of Organic Material on Magnetoresistance in Electron-doped Double Perovskite
}

\author{
Yu Liu \\ Henan Normal University \\ Yan-Ming Zhang \\ Henan Normal University \\ Jin-Feng Wang \\ Henan Normal University
}

Ya Fang Li ( $\nabla$ missli1129@163.com )

Henan Normal University https://orcid.org/0000-0002-3325-9129

\section{Research Article}

Keywords: La0.5Sr1.5FeMoO6 double perovskite, Inorganic/organic composite, Magnetoresistance, Grain boundary

Posted Date: March 18th, 2021

DOI: https://doi.org/10.21203/rs.3.rs-297068/v1

License: (1) (1) This work is licensed under a Creative Commons Attribution 4.0 International License. Read Full License 
2 previous works confirmed that, a remarkable enhanced LFMR was observed in $\mathrm{Sr}_{2} \mathrm{FeMoO}_{6}$ by modifying the grain boundary with insulating organic small molecules (glycerin, $\mathrm{CH}_{2} \mathrm{OHCHOHCH}_{2} \mathrm{OH}$ ). However, in this work, modifying the grain boundary strength of the $\mathrm{La}_{0.5} \mathrm{Sr}_{1.5} \mathrm{FeMoO}_{6}$ with the insulating organic macromolecules (oleic acid, $\mathrm{CH}_{3}\left(\mathrm{CH}_{2}\right)_{7} \mathrm{CH}=\mathrm{CH}\left(\mathrm{CH}_{2}\right)_{7} \mathrm{COOH}$ ) or small molecules (glycerin), both of them have negligible functions on the magnetoresistance behavior in $\mathrm{La}_{0.5} \mathrm{Sr}_{1.5} \mathrm{FeMoO}_{6}$. Contrary to the magnetoresistance property. Based on the above experimental results and the related works, it is proposed that, maintaining high spin polarization of the carriers at the Fermi level and improving the tunneling process across the grain boundary by using the suitable organic materials are decisive factors for optimizing the magnetoresistance behavior in the similar electron-doped double perovskites.

21 Key words: $\mathrm{La}_{0.5} \mathrm{Sr}_{1.5} \mathrm{FeMoO}_{6}$ double perovskite; Inorganic/organic composite; Magnetoresistance;

\section{double perovskite}

\author{
*Corresponding author
}

College of Physics, National Demonstration Center for Experimental Physics Education, Henan Normal University and Henan Key Laboratory of Photovoltaic Materials, Xinxiang 453007, China glycerin-modified $\mathrm{Sr}_{2} \mathrm{FeMoO}_{6}, \mathrm{Sr}_{2} \mathrm{FeMoO}_{6} /$ oleic acid composites don’t exhibit an obviously increased

Grain boundary 


\section{Declarations}

24 Funding This work was supported by fund from the Natural Science Foundation of China (U1504107).

25 Conflict of interest The authors declare that there is no conflict of interest.

\section{Relevance Summary}

27 1. The Curie temperature of electron-doped $\mathrm{Sr}_{2} \mathrm{FeMoO}_{6}$ can be optimized significantly but always accompanying an almost absent of low-field magnetoresistance, which is unfavorable to applications in the magnetoresistive devices operated at room-temperature.

30 2. In this work, the transport and magnetic properties of $\mathrm{La}_{0.5} \mathrm{Sr}_{1.5} \mathrm{FeMoO}_{6}$ modified by organic 31 materials are discussed systematically. And we may provide an effective method to solve the 32 problem. 


\section{Introduction}

Due to its a half-metallic property with the $100 \%$ spin-polarization at the Fermi level, a high Curie temperature $\left(\mathrm{T}_{\mathrm{C}}\right.$ ) of $\sim 415 \mathrm{~K}$ and remarkable large low-filed magnetoresistance (LFMR) behavior, double perovskite $\mathrm{Sr}_{2} \mathrm{FeMoO}_{6}(\mathrm{SFMO})$ has been paid a great deal of attention in views of its critical fundamental investigation values and immense potential technological applications for spintronic and magnetoresistive devices operated at room temperature [1].

It is well known that in an ideal SFMO double perovskite structure, the $\mathrm{FeO}_{6}$ and $\mathrm{MoO}_{6}$ octahedral arrange alternatively along the three axes of tetragonal structure with the Sr cations occupy the voids between them. Strong antiferromagnetic correlation exists between the localized magnetic moments $\left(\mathrm{Fe}^{3+}: 3 \mathrm{~d}^{5}\right)$ and the delocalized electron $\left(\mathrm{Mo}^{5+}: 4 \mathrm{~d}^{1}\right)$ in a double-exchange-like type, the magnetic coupling induces a ferrimagnetic state with an ideal saturated magnetization $\left(\mathrm{M}_{\mathrm{S}}\right)$ of $4 \boldsymbol{\mu}_{\boldsymbol{B}}$ per formula unit [2-7]. The strength of magnetic coupling in SFMO double perovskite is mainly controlled by the carrier density at the Fermi level, so it indicates that doping electrons in the conduction band is an effective way to enhance $T_{C}[8-14]$. This point was confirmed by a substantial enhancement of $T_{C}$ more than $80 \mathrm{~K}_{\text {in }} \mathrm{La}_{\mathrm{x}} \mathrm{Sr}_{(2-\mathrm{x})} \mathrm{FeMoO}_{6}[9]$ and $\left(\mathrm{Ba}_{0.8} \mathrm{Sr}_{0.2}\right)_{2-\mathrm{x}} \mathrm{La}_{\mathrm{x}} \mathrm{FeMoO}_{6}$ materials [15]. Obviously, the celebrated strategy increases the operating temperature range of the electromagnetic applications in SFMO double perovskite. However, the increased $\mathrm{T}_{\mathrm{C}}$ in electron-doped double perovskites always accompany with a strong suppression on MR effect $[9,15,16]$. This phenomenon seriously affects functional properties of materials and constrains the technical application. Hence, it is necessary and meaningful to improve the MR effect in electron-doping SFMO system.

As a fact, a remarkable large MR effect can be observed in polycrystalline SFMO ceramics, but it is almost absent in SFMO single crystals or epitaxial films [17-19]. This suggests that the LFMR of 
55 SFMO is a type of tunneling magnetoresistance, the transport process is related with the spin-dependent scattering occurred at magnetic domain boundaries, so the existence of grain boundary in SFMO is vital

57 for magnetoresistance [1]. Previous research proved that LFMR could be improved by enhancing the GB

58 strength in many methods, such as adding the second phase in GB [20, 21], slightly oxidizing GB [22,

$5923]$, reducing the grain size $[24,25]$ and dispersing the grain uniformly [26]etc. It should be noted that there are three common points in these methods. First, the increment of LFMR value always accompanies

61 with the enhancement of resistivity. Actually, a function of $\boldsymbol{\rho} \propto \boldsymbol{\operatorname { e x p }}(\boldsymbol{\gamma} \boldsymbol{} \sqrt{\Delta})$ was proposed to express the strength of the GB insulating barriers. From this, the GB strength is directly measured by $\boldsymbol{\rho}$ [27].

63 Second, the increment of LFMR value always with the decrement of magnetization. Moreover, the experiment process of above methods is relatively complex. Therefore, it is necessary to establish a facile method which can maintain the magnetization while improve the LFMR value of SFMO. In our previous research, the SFMO ceramic was soaked in organic matter, by the method directly, the resistivity was increased about 500 times and the LFMR value was effectively improved up to $-29.5 \%$ at $10 \mathrm{~K}$ [28]. Based on the achievement, we infer that the method of preparing $\mathrm{La}_{0.5} \mathrm{Sr}_{1.5} \mathrm{FeMoO}_{6}$ (LSFMO)/organic matter composites could be used to eliminate the negative effect resulting from electrons doping in SFMO ceramics and then optimize the LFMR effect while ensuring the magnetization. The organic matters of oleic acid $\left(\mathrm{CH}_{3}\left(\mathrm{CH}_{2}\right)_{7} \mathrm{CH}=\mathrm{CH}\left(\mathrm{CH}_{2}\right)_{7} \mathrm{COOH}\right)$ and glycerin $\left(\mathrm{CH}_{2} \mathrm{OHCHOHCH}{ }_{2} \mathrm{OH}\right)$ were used as the modifying material in this work. The oleic acid has a longchain structure with the molecular weight of 282.45 and the glycerin has a short-chain structure with the

74 molecular weight of 92.09. It is obvious that the former has the more excellent insulation. prepared to investigate the effect of organic materials on the magnetoresistance behavior in electron- 
doped double perovskite. The structure, magnetization, electrical resistivity and magnetoresistance of composites were investigated systematically and comparatively. The main results indicate that neither of the MR behavior in the three experiments has been optimized effectively. It proves that it is critical to select a suitable organic material to modify GB while maintain the high spin polarization of the carriers at the Fermi level in the electron-doped double perovskite.

\section{Experimental}

\subsection{The method of Experiment I ( $\mathrm{La}_{0.5} \mathrm{Sr}_{1.5} \mathrm{FeMoO}_{6} /$ oleic acid composite)}

$\mathrm{La}_{0.5} \mathrm{Sr}_{1.5} \mathrm{FeMoO}_{6}$ ceramics were synthesized by a conventional solid-state reaction method. First, the appropriate amounts of analytic grade $\mathrm{La}_{2} \mathrm{O}_{3}, \mathrm{SrCO}_{3}, \mathrm{Fe}_{2} \mathrm{O}_{3}$ and $\mathrm{MoO}_{3}$ powders were weighted, ground $3 \mathrm{~h}$ in an agate mortar and sintered at $900{ }^{\circ} \mathrm{C}$ for $10 \mathrm{~h}$ in air. The sintered powder was ground again and then pressed into disks. After that, these disks were annealed at $1200{ }^{\circ} \mathrm{C}$ for $12 \mathrm{~h}$ in the $5 \%$ $\mathrm{H}_{2} / 95 \%$ Ar reducing atmosphere. Pure LSFMO powder was collected after triturating these annealed disks.

To modify the GB strength, the as-prepared pure LSFMO powder was post-treated as follows: first, different volume proportions of the oleic acid $\left(\mathrm{CH}_{3}\left(\mathrm{CH}_{2}\right)_{7} \mathrm{CH}=\mathrm{CH}\left(\mathrm{CH}_{2}\right)_{7} \mathrm{COOH}, V_{l}\right)$ and alcohol $\left(V_{2}\right)$ were fully stirred to form the mixed solution $\left(V=0,0.1,0.2,0.3,0.4,0.5, \boldsymbol{V}=\boldsymbol{V}_{\mathbf{1}} / \boldsymbol{V}_{\mathbf{1}}+\boldsymbol{V}_{\mathbf{2}}\right)$. For each treatment, $20 \boldsymbol{\mu} \boldsymbol{L}$ of the mixed solution was pipetted and added into $0.2 \mathrm{~g}$ as-prepared LSFMO powder. After six pipetting times, the formed organic/inorganic composite was thoroughly admixed and pressed into disk, then conserved at room temperature (RT). The collected composites were labeled as C1-C6, respectively.

\subsection{The method of Experiment II ( $\mathrm{La}_{0.5} \mathrm{Sr}_{1.5} \mathrm{FeMoO}_{6} /$ glycerin composite)}

For contrasting with Experiment I, in this experiment, the modificatory factor oleic acid was 
99 substituted by glycerin $\left(\mathrm{CH}_{2} \mathrm{OHCHOHCH}_{2} \mathrm{OH}\right)$. Pure LSFMO powder (in Experiment I) was pressed

100 into disks and the mass of every disk was $0.2 \mathrm{~g}$. To control the participating content of glycerin at GB,

101 the disks were soaked in the isometric mixed reagent of glycerin and alcohol under the protection of $\mathrm{N}_{2}$,

102 and kept static at room temperature for 5 and 15 days. Subsequently, the disks were taken out and gently

103 washed three times by alcohol, dried in vacuum condition at RT. The collected disks were labeled as C7

104 (5 days) and C8 (15 days).

\section{$105 \quad 2.3$ The method of Experiment III $\left(\mathrm{Sr}_{2} \mathrm{FeMoO}_{6} /\right.$ oleic acid composite)}

106 The modified object LSFMO was substituted by pure SFMO. Specifically, the suitable amounts of

107 analytic grade $\mathrm{SrCO}_{3}, \mathrm{Fe}_{2} \mathrm{O}_{3}$ and $\mathrm{MoO}_{3}$ powders were weighted, ground and sintered at $900{ }^{\circ} \mathrm{C}$ for $10 \mathrm{~h}$

108 in air. Then the sintered powder was ground again for $3 \mathrm{~h}$ and annealed at $1200^{\circ} \mathrm{C}$ for $12 \mathrm{~h}$ in $5 \% \mathrm{H}_{2} / 95 \%$

109 Ar reducing atmosphere. To modify the GBs, $0.2 \mathrm{~g}$ of SFMO powder was mixed homogeneously with

$11020 \boldsymbol{\mu} \boldsymbol{L}$ isopyknic mixed reagent of oleic acid and alcohol solution here. After completely admixing, the

111 composite was pressed into disk and conserved at RT. The collected disk was labeled as C9.

113 Discover. The microstructure of the samples was examined through high resolution field emission

114 scanning electron microscope (FESEM). The C element's distribution was carried out using energy

115 dispersive X-ray spectroscopy (EDS) coupled with FESEM instrument. The magnetization and magnetic

116 transport data of all samples (C1-C9) were carried out by a physical property measurements system

117 (PPMS Quantum Design). 


\section{Results and discussion} and EDS measurements, it is reasonable to conclude that the physisorption of oleic acid in LSFMO grain

The crystal structure of all prepared polycrystalline samples was detected by the X-ray diffraction (Fig. 1). From the picture, it can be clearly seen that all the ceramics were well consistent with tetragonal space group of I4/m. Neither XRD peak shifting nor other impurities can be observed in the composites. It manifests that the organic molecules are mainly located at grain boundaries and have any effect on the structure of original samples. Otherwise, it can also be interpreted as there was negligible or even no chemical reaction between them. $\mathrm{SrMoO}_{4}$ as a common second phase exists in SFMO double perovskite can be obtained by oxidation [23, 29] or sonication process [30] in experiments. However, such a secondary phase is not shown up in this work which is evidenced by XRD results. The discrepancies between the present study and the previous one [29] may result from the different experimental manipulations, like the prepared method of samples and the content of the solvent in organic matter.

Fig.2 shows the FESEM images for pristine LSFMO (C1) and LSFMO/oleic acid composites (C2C6). The FESEM images show the grain size about $2.5 \mu \mathrm{m}$ in all composites. The phenomenon that LSFMO grains with small size are inlaid in measured areas can be observed in these images. This is attributed to the physical mixing process. In this process, the LSFMO's binding force forming in the annealing time is destroyed by the external pressure and then grains are dispersed, although they are subsequently pressed into disks again, it cannot distribute uniformly like the annealed samples. The $\mathrm{C}$ element' distributions in these samples measured with EDS are shown in the insets in Figure 2. It can be observed that the content of oleic acid increases with the increment of the $V$ values. Based on the FESEM regions might be the dominant states, which is also confirmed by the XRD analysis. 


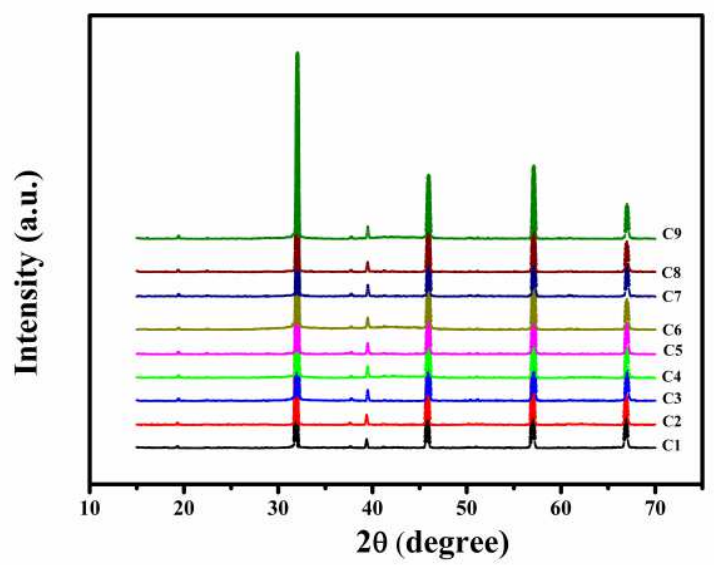

Fig. 1 The XRD patterns of all prepared ceramics (C1-C9)
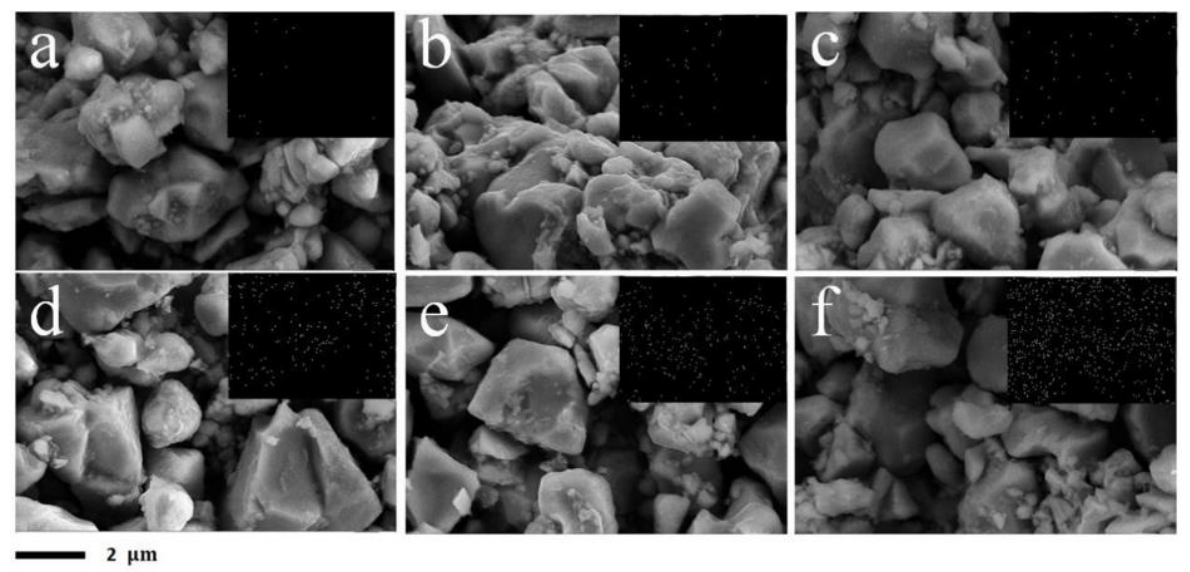

141 solution $(V=0,0.1,0.2,0.3,0.4,0.5)$ composites were measured at $50 \mathrm{~K}$ and $300 \mathrm{~K}$, shown in Fig. 3 and 4 respectively. Evidently, all the samples show the ferromagnetic nature with a well-saturated feature

Fig. 2 FESEM images of oleic acid/La0.5 $\mathrm{Sr}_{1.5} \mathrm{FeMoO}_{6}$ composites, (a-f) correspond to C1-C6 samples. The insets are the corresponding $\mathrm{C}$ element mappings

The magnetic hysteresis loops of LSFMO with different volume proportions of oleic acid/alcohol

147 between $-2.5 \mathrm{~T}$ and $+2.5 \mathrm{~T}$. The mass differences of the pure LSFMO with LSFMO/oleic acid composites

148 are provided in Table 1. It can be observed that the mass of organic matter that entered into LSFMO

149 grain boundaries is too little and this extra mass has negligible effect when we calculate the magnetization

150 values. With the oleic acid content increasing, the $\mathrm{M}_{\mathrm{S}}$ values at $50 \mathrm{~K}$ are respectively $1.46,1.41,1.68$,

$151 \quad 1.33,1.32$ and $1.47 \boldsymbol{\mu}_{B} / \boldsymbol{f} . \boldsymbol{u}$. of the corresponding C1-C6 composite samples, which suggests the organic 
153 acid molecules segregate at the grain boundaries, they have no obvious effect on the LSFMO

154 ferromagnetic domains. An analogous behavior appears in the M-H curves at $300 \mathrm{~K}$ (Fig.4).

155

156

157

158

159

160

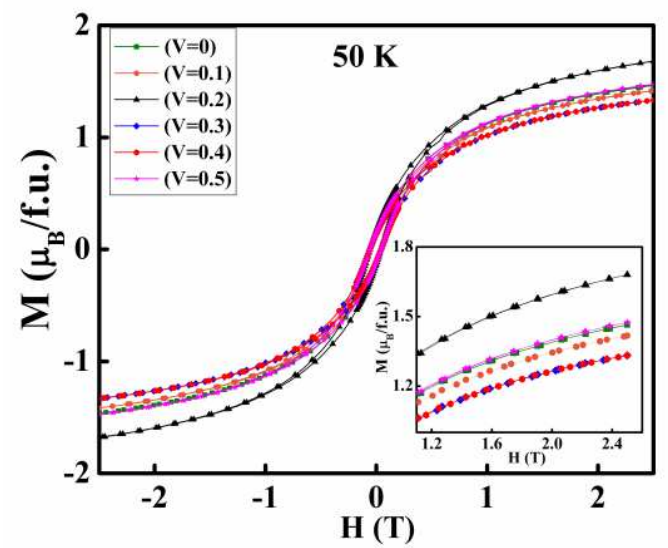

Fig. 3 The magnetization versus magnetic field (M-H) curves measured at $50 \mathrm{~K}$ for Experiment I. The inset is the locally enlarged M-H curves

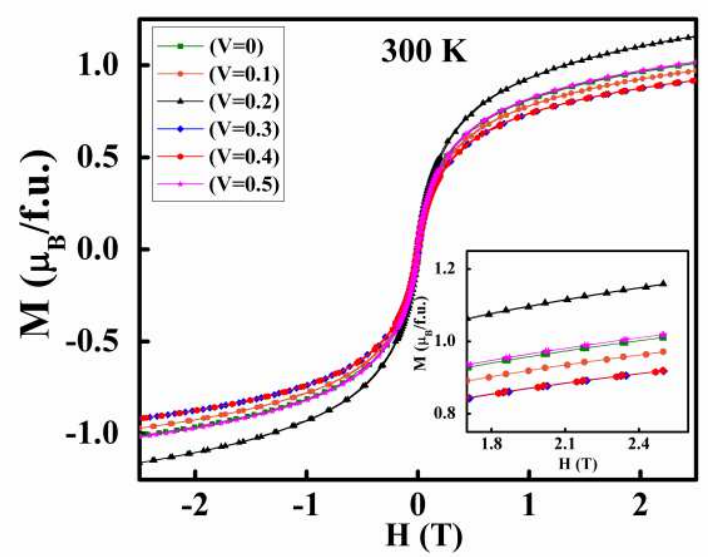

Fig. 4 The magnetization versus magnetic field (M-H) curves measured at $300 \mathrm{~K}$ for C1-C6 in Experiment I.

The inset is the locally enlarged M-H curves

161 Table 1 The mass differences of the pure LSFMO with LSFMO/oleic acid composites

\begin{tabular}{ccccccc}
\hline Sample & $\mathrm{C} 1$ & $\mathrm{C} 2$ & $\mathrm{C} 3$ & $\mathrm{C} 4$ & $\mathrm{C} 5$ & $\mathrm{C} 6$ \\
\hline $\begin{array}{c}\text { Mass before } \\
\text { soaking }(\mathrm{g})\end{array}$ & 0.2000 & 0.2000 & 0.2000 & 0.2000 & 0.2000 & 0.2000 \\
\hline $\begin{array}{c}\text { Mass after soaking } \\
(\mathrm{g})\end{array}$ & 0.2000 & 0.2002 & 0.2008 & 0.2011 & 0.2027 & 0.2036 \\
\hline $\begin{array}{c}\text { Mass difference } \\
(\%)\end{array}$ & $0 \%$ & $0.10 \%$ & $0.40 \%$ & $0.55 \%$ & $1.35 \%$ & $1.80 \%$ \\
\hline
\end{tabular}




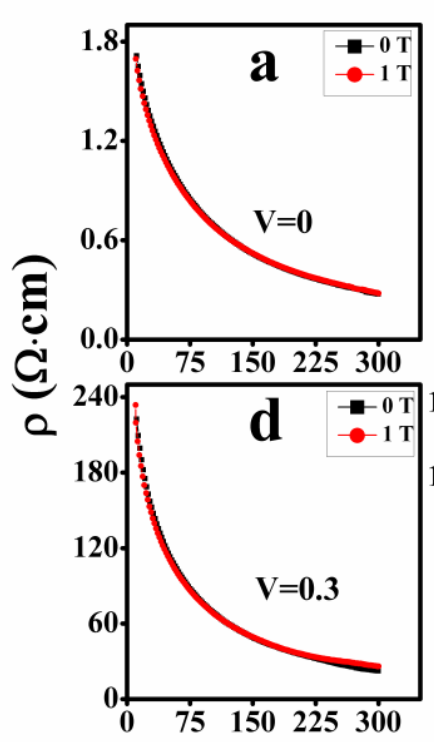

162

163

164

165

166

167

168

169

170

171 (without extra field) of C1-C6 at $50 \mathrm{~K}$ and $300 \mathrm{~K}$ are shown (Fig. 6) In this picture, values of C6

172

composite are $138.8 \Omega \mathrm{cm}$ and $27.5 \Omega \mathrm{cm}$ at $50 \mathrm{~K}$ and $300 \mathrm{~K}$ respectively, exhibiting $\sim 130$ times and

173

174

175 effectively weak the tunneling conduction ability of charger carriers when they across the grain 176
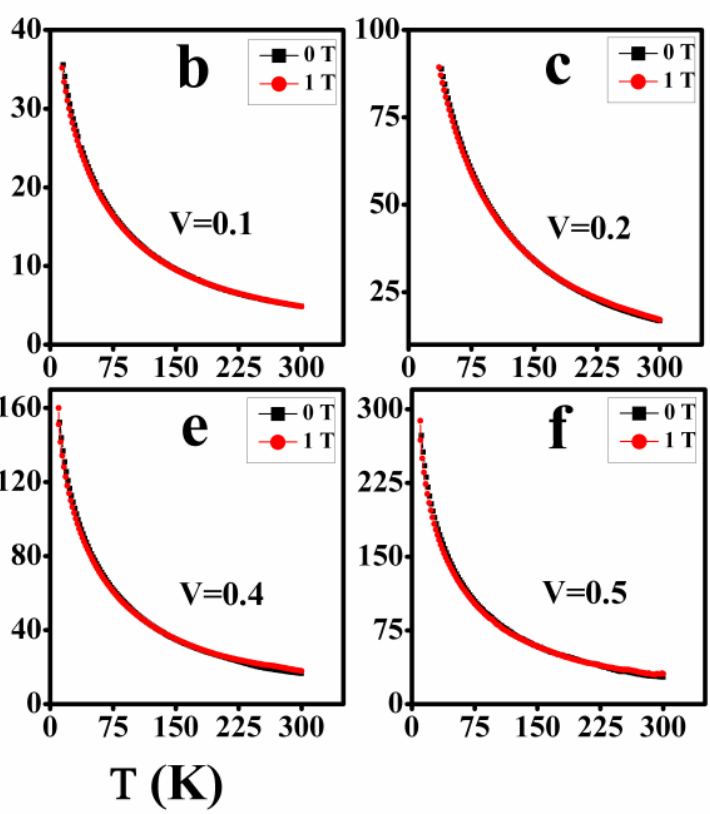

Fig. 5 Temperature dependent resistivity curves for C1-C6 (a-f) measured at $10 \mathrm{~K}-300 \mathrm{~K}$ with $0 \mathrm{~T}$ and $1 \mathrm{~T}$ extra field

Fig. 5 shows the temperature dependent resistivity curves for $\mathrm{C} 1-\mathrm{C} 6$ composites with $0 \mathrm{~T}$ and $1 \mathrm{~T}$

extra field. All the ceramics exhibit a semiconductor-like behavior in the range of investigating. In this

system, it can be observed a systematic enhancement in resistivity over the whole temperature range of

10-300 K while adding the extra amount of oleic acid without reducing the SFMO content. The measured

curves with $0 \mathrm{~T}$ and $1 \mathrm{~T}$ are almost overlapped in this figure, it suggests extra field has no obvious

influence on resistance property. In order to describe resistivity more directly, the resistivity values

$\sim 100$ times of $\mathrm{C} 1(1.0661 \Omega \mathrm{cm}$ at $50 \mathrm{~K}, 0.27 \Omega \mathrm{cm}$ at $300 \mathrm{~K})$. The variation of resistivity implies that

the charge transport is significantly influenced by the nature of GB, it can be explained by that oleic acid

boundaries. The jump points in $\mathrm{C} 5$ composite $(V=0.4)$ may result from the preparation process. 


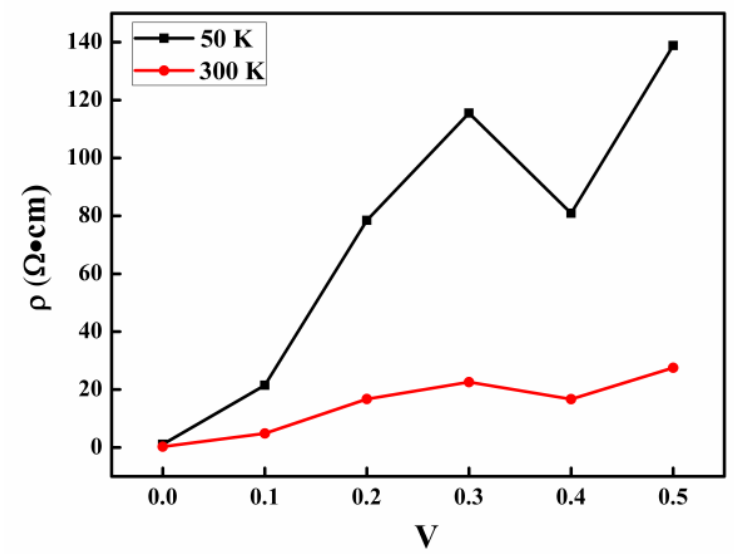

177

178

179

180

181

182

183

184

185

186

187

188

189

190

191

Fig. 6 The resistivity-volume proportions curves of samples (C1-C6) at $50 \mathrm{~K}$ and $300 \mathrm{~K}$ with zero-field

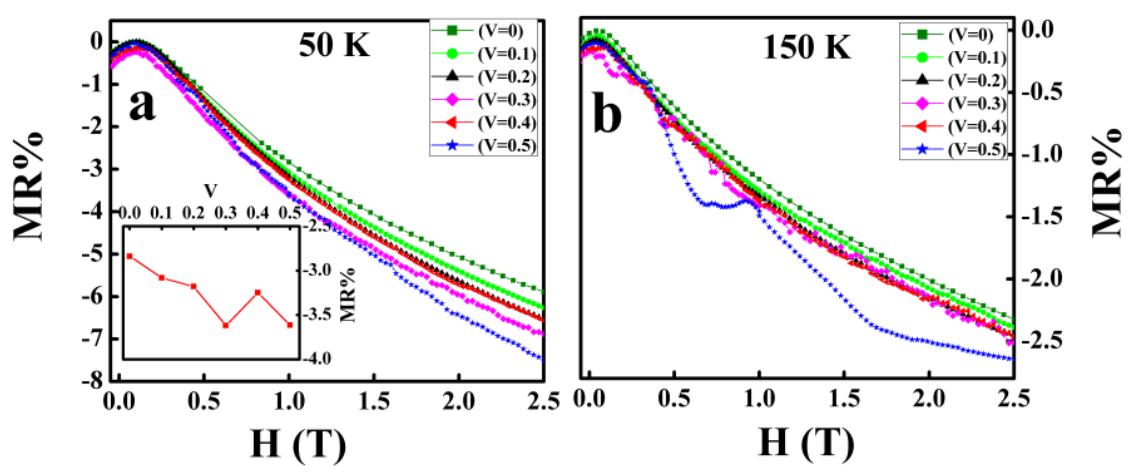

Fig. 7 MR\% versus applied magnetic field plots of $\mathrm{La}_{0.5} \mathrm{Sr}_{1.5} \mathrm{FeMoO}_{6}$ with different volume proportions of oleic acid/alcohol contents $(V=0,0.1,0.2,0.3,0.4,0.5)$ measured at $50 \mathrm{~K}$ (a) and $150 \mathrm{~K}$ (b). The inset in (a) was the volume proportions dependent MR at $1 \mathrm{~T}$

MR\% versus applied magnetic field (MR\%-H) curves for C1-C6 at $50 \mathrm{~K}$ and $150 \mathrm{~K}$ were depicted

in Fig. 7a and Fig. 7b, respectively. The MR\% is defined here as $\boldsymbol{M} \boldsymbol{R} \%=\left(\boldsymbol{\rho}_{\boldsymbol{H}}-\boldsymbol{\rho}_{\mathbf{0}}\right) / \boldsymbol{\rho}_{\mathbf{0}} \times \mathbf{1 0 0} \%$,

where $\boldsymbol{\rho}_{\boldsymbol{H}}$ and $\boldsymbol{\rho}_{\mathbf{0}}$ are the values of resistivity with and without a magnetic field. From figure 7, the MR\%

value is slightly enhanced with the organic matter content, but the enhancement is negligible when

compare with other researches [28-32]. From the inset in Fig. 7a, the LFMR $(\mathrm{H}=1 \mathrm{~T})$ value of $\mathrm{C} 1$

composite at $50 \mathrm{~K}$ is $-2.8 \%$ and it gets to the maximum $-3.6 \%$ of $\mathrm{C} 6$, it just improves $0.8 \%$. Moreover,

the same phenomenon occurred at $150 \mathrm{~K}$, depicted in Fig. $7 \mathrm{~b}$. These data can strongly prove that the method of modifying LSFMO by organic matter is not available to improve LFMR response. This result violates the previous inference which is the stronger GB strength portend higher MR. To make our result 
193 Unfortunately, according to the analysis with Experiment I, we conclude that the method is not workable.

Fig. 8 shows the magnetization versus temperature plots of $\mathrm{C} 1-\mathrm{C} 6$ composite samples that measured from $300 \mathrm{~K}$ to $450 \mathrm{~K}$. Almost samples undergo a ferromagnetic to paramagnetic transition at the same temperature $(420 \mathrm{~K})$. By adding the oleic acid molecules in GB, there is no obvious change in the magnetic transition temperature of samples. This is yet another confirmation that the organic matter is not beneficial to enhance the ferromagnetic coupling strengths of the LSFMO material.

It is expected that, the insulating big molecules (oleic acid) located at GBs of LSFMO double perovskite can increase the tunneling probability of spin electrons, and thus enhance the LFMR behavior.

However, a remarkable LFMR enhancement in SFMO modified with small molecules (glycerin) was observed in our previous study, in which the LFMR of SFMO/glycerin composite was more than 2 times larger than that of the pure SFMO [28]. Therefore, we designed Experiment II (LSFMO/glycerin) to further study.

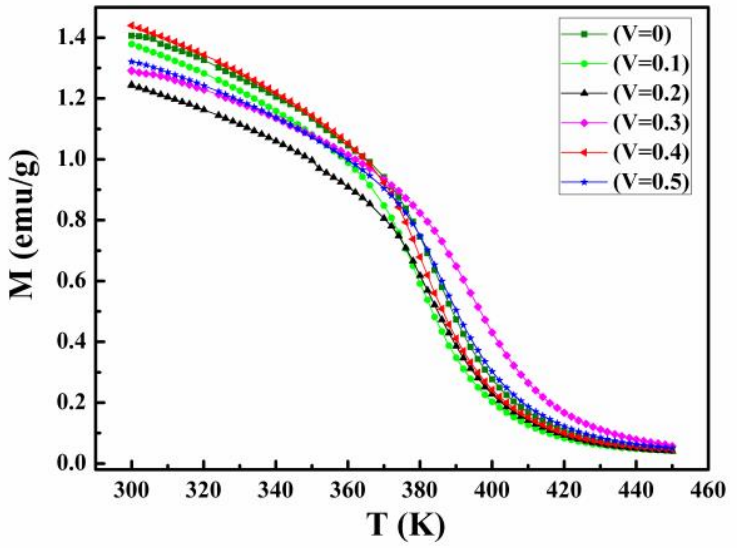

Fig. 8 Magnetization-temperature (M-T) curves for C1-C6 measured from $300 \mathrm{~K}$ to $450 \mathrm{~K}$ 


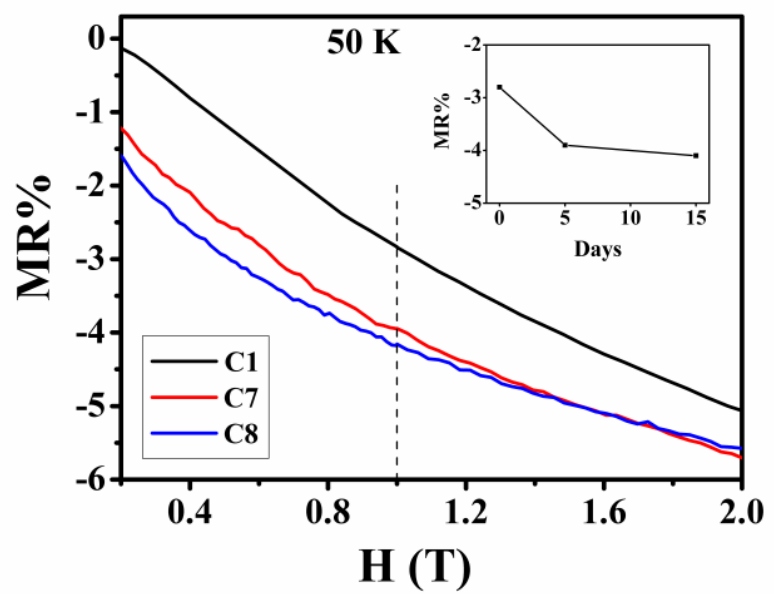

Fig. $9 \mathrm{MR} \%$ versus applied magnetic field of $\mathrm{C} 1, \mathrm{C} 7$ and $\mathrm{C} 8$ measured at $50 \mathrm{~K}$ (Experiment II). The inset is the obviously that the LFMR effect of LSFMO/glycerin composite cannot also be optimized very well, which has a sharp contrast with SFMO/glycerin [28]. The difference between them may lie in that, La

216 electrons doped at $\mathrm{Sr}$ sites leads to two effects: one is band-filling effect, and the other is the increased $\mathrm{Fe} / \mathrm{Mo}$ anti-site defects concentration, both of them can decrease the amounts of spin-polarized electrons

218 that tunneling across GB. So, we can conclude that maintaining high spin polarization of the carriers at

219 the Fermi level is a crucial factor to obtain an excellent MR behavior in similar electron-doped double 220 perovskite.

221 Based on the experiments, we noticed that it is necessary to maintain a high spin polarization of

222 carriers to obtain a large MR effect. In Experiment III, we prepared the SFMO with a high Fe/Mo

223 ordering degree to ensure a high spin polarization of carriers at the Fermi level, and then fabricate it with the oleic acid molecule. 


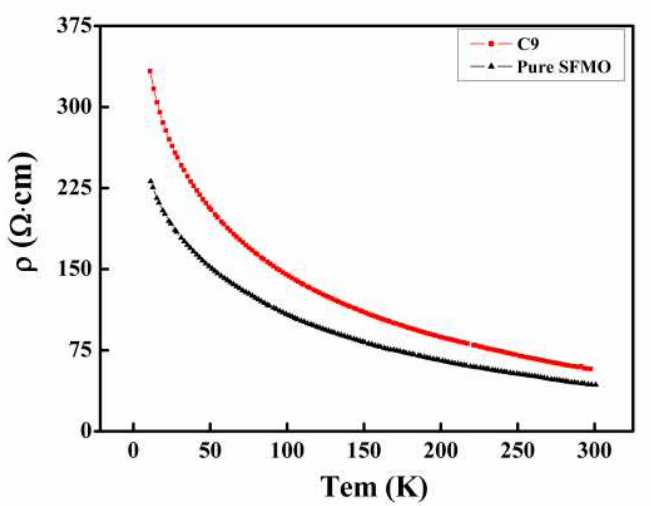

Fig. 10 Resistivity versus temperature curves for pure SFMO and C9 at $10 \mathrm{~K}-300 \mathrm{~K}$ with zero-field

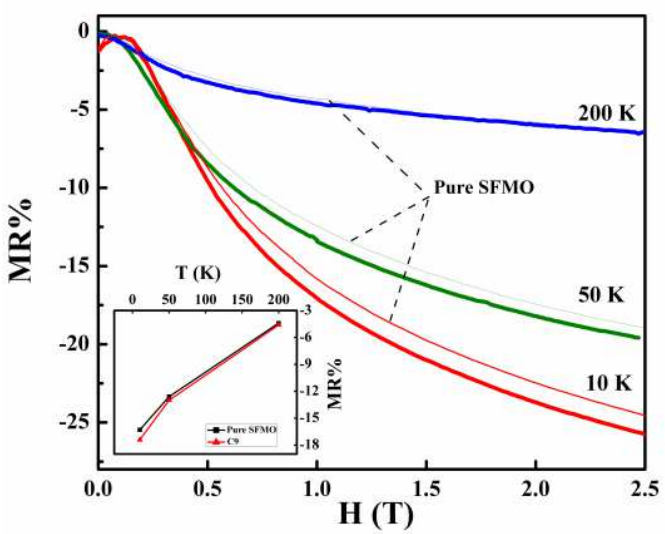

Fig. 11 MR\% versus applied magnetic field of pure SFMO sample (thin lines) and C9 (thick lines) measured at $10 \mathrm{~K}, 50 \mathrm{~K}$ and $200 \mathrm{~K}$. The inset in figure is the measured temperature dependent low field magnetoresistance at $1 \mathrm{~T}$ for the two samples

232 oleic acid in the whole temperature range, similarly with the previous statement. The MR of the pure SFMO (thin lines) and C9 composite (thick lines) were measured at $10 \mathrm{~K}, 50 \mathrm{~K}$ and $200 \mathrm{~K}$, shown in

Figure 11. As the picture presents, the LFMR of pure SFMO at $1 \mathrm{~T}$ are $-16.3 \%,-12.6 \%,-4.4 \%$ respectively and $-17.4 \%,-13.0 \%,-4.6 \%$ for $\mathrm{C} 9$ in $10 \mathrm{~K}, 50 \mathrm{~K}$ and $200 \mathrm{~K}$. It is obvious that the SFMO modified by oleic acid still cannot effectively optimize the LFMR. Otherwise, this behavior is in a sharp contrast with the SFMO/glycerin work [28]. Therefore, we noticed that the different results may due to the different molecular structure and insulation properties of oleic acid and glycerin. 
magnetic materials [33-36]. In these works, the chemical bonding between organic molecules and

241 ferromagnetic grains is the decisive factor for the enhancement of LFMR. Oleic acid is recognized as an

242 efficient barrier when it closely contacts with the $\mathrm{Fe}_{3} \mathrm{O}_{4}$ or $\mathrm{La}_{0.7} \mathrm{Sr}_{0.3} \mathrm{MnO}_{3}$ grains with a single molecular

243 layer. But this phenomenon has not happened in our present work. It may derive from that oleic acid

244 molecule is physically bonded with SFMO grains.

An efficient optimization of LFMR have been observed in SFMO/glycerin [28], which has a sharp

246 contrast with SFMO/oleic acid. The reasons maybe lie in the different molecular structure of the organic matter and bonding strength between organic molecules and SFMO grain boundary. As for SFMO/oleic

248 acid, it is difficult to form a single layer between two grains due to big molecule oleic acid and different

249 preparation process compared with $\mathrm{Fe}_{3} \mathrm{O}_{4}$ or $\mathrm{La}_{0.7} \mathrm{Sr}_{0.3} \mathrm{MnO}_{3}$ grains, thus the stronger energy barrier

250 between oleic acid big molecule and SFMO grain will weak the spin injection efficiency and therefore

251 suppress to LFMR effect. But in the SFMO/glycerin system, compared with oleic acid, glycerin has a

252 smaller molecular size and three hydroxyl groups, leads to a relative strong bonding strength with the

253 SFMO interfacial grain. Therefore, glycerin is beneficial to spin injection and the improvement of LFMR

254 effect.

255 Based on the analysis, we conclude that oleic acid can be recognized as a suitable transport medium

256 when it chemically bonds to ferromagnetic grain. But when it bonds in physically, like the present work,

257 oleic acid cannot optimize efficiently LFMR of the SFMO. In this case, it's available to select the smaller

258 organic molecule with many hydroxyl groups to modify GB to enhance LFMR in the similar double 259 perovskite materials. 


\section{Conclusion}

$\mathrm{La}_{0.5} \mathrm{Sr}_{1.5} \mathrm{FeMoO}_{6} /$ oleic acid composite was prepared to expect to improve the magnetoresistance of

$\mathrm{La}_{0.5} \mathrm{Sr}_{1.5} \mathrm{FeMoO}_{6}$ electron-doped double perovskite in Experiment I, but the result shows that the

modifying treatment is noneffective. In order to make it clear, $\mathrm{La}_{0.5} \mathrm{Sr}_{1.5} \mathrm{FeMoO}_{6} /$ glycerin composite

(Experiment II) and $\mathrm{Sr}_{2} \mathrm{FeMoO}_{6}$ /oleic acid composite (Experiment III) were designed and the MR

behavior were detailedly disscussed respectively. Contrary to the glycerin-modified $\mathrm{Sr}_{2} \mathrm{FeMoO}_{6}$ which

has made a remarkable enhanced LFMR in our previous works, both of the composites in Experiment II

works, we conclude the invalid MR effect on $\mathrm{La}_{0.5} \mathrm{Sr}_{1.5} \mathrm{FeMoO}_{6} /$ oleic acid composite results from two

respects: the lower spin polarization of carriers at the Fermi level and the weaker spin injection efficiency

in GB derived from the oleic acid macromolecule. Maybe our work provides a direction for the MR

\section{References}

275 [1] K.I. Kobayashi, T. Kimura, H. Sawada, K. Terakura, Y. Tokura, Nature 395, 677 (1998)

276 [2] L. Balcells, J. Navarro, M. Bibes, A. Roig, B. Martínez, J. Fontcuberta, Appl. Phys. Lett. 78, 781 277 (2001)

278 [3] J. Navarro, J. Nogués, J.S. Muñoz, J. Fontcuberta, Phys. Rev. B 67, 174416 (2003)

279 [4] Z. Wang, A.H. Tavabi, L. Jin, J. Rusz, D. Tyutyunnikov, H. Jiang, Y. Moritomo, J. Mayer, R.E. Dunin280 Borkowski, R. Yu, J. Zhu, X. Zhong, Nat. Mater. 17, 221 (2018)

281 [5] I. Hussain, M.S. Anwar, S.N. Khan, J.W. Kim, K.C. Chung, B.H. Koo, J. Alloys Compd. 694, 815 282 (2017)

283 [6] S. Varaprasad, K. Thyagarajan, Y. Markandeya, K. Suresh, G. Bhikshamaiah, J. Mater. Sci. : Mater. 284 Electron. 29, 13606 (2018)

285 [7] L.D. Hien, N.P. Duong, L.N. Anh, T.T. Loan, S. Soontaranon, A. de Visser, J. Alloys Compd. 793, 286375 (2019)

287 [8] M. Tovar, M.T. Causa, A. Butera, J. Navarro, B. Martínez, J. Fontcuberta, M.C.G. Passeggi, Phys. 
Rev. B 66, 024409 (2002)

[9] J. Navarro, C. Frontera, L. Balcells, B. Martínez, J. Fontcuberta, Phys. Rev. B 64, 092411 (2001)

[10] J. Fontcuberta, D. Rubi, C. Frontera, J.L. García-Muñoz, M. Wojcik, E. Jedryka, S. Nadolski, M.

291 Izquierdo, J. Avila, M.C. Asensio, J. Magn. Magn. Mater. 290-291, 974 (2005)

292 [11] C. Frontera, D. Rubi, J. Navarro, J.L. García-Muñoz, J. Fontcuberta, C. Ritter, Phys. Rev. B 68, $293012412(2003)$

294 [12] Q. Zhang, Z.F. Xu, H.B. Sun, X. Zhang, H. Wang, G.H. Rao, J. Alloys Compd. 745, 525 (2018)

295 [13] J.F. Wang, T.F. Shi, Z.T. Zhuang, Q.Q. Gao, Y.M. Zhang, RSC Adv. 8, 29071 (2018)

[14] Imad Hussain, M.S. Anwar, S.N. Khan, Jong Woo Kin, Kook Chae Chung, Bom Heun Koo, J. Alloys

297 Compd. 694, 815 (2017)

298 [15] D. Serrate, J.M. De Teresa, J. Blasco, M.R. Ibarra, L. Morellón, C. Ritter, Appl. Phys. Lett. 80, 4573 $299 \quad$ (2002)

300 [16] D. Rubi, C. Frontera, J. Nogués, J. Fontcuberta, J. Phys.: Condens. Matter 16, 3173 (2004)

301 [17] C.L. Yuan, S.G. Wang, W.H. Song, T. Yu, J.M. Dai, S.L. Ye, Y.P. Sun, Appl. Phys. Lett. 75, 3853 302 (1999)

303 [18] S.R. Shinde, S.B. Ogale, R.L. Greene, T. Venkatesan, K. Tsoi, S.W. Cheong, A.J. Millis, J. Appl. 304 Phys. 93, 1605 (2003)

305 [19] Y. Tomioka, T. Okuda, Y. Okimoto, R. Kumai, K. Kobayashi, Y. Tokura, Phys. Rev. B 61, 422 (2000)

306 [20] N. Kumar, G. Khurana, A. Gaur, R.K. Kotnala, J. Appl. Phys. 114, 053902 (2013)

307 [21] J. Zhang, W.J. Ji, J. Xu, Z.B. Gu, Y.B. Chen, S.T. Zhang, J. Phys.: Condens. Matter 31, 225001 308 (2019)

309 [22] D. Niebieskikwiat, A. Caneiro, R.D. Sánchez, J. Fontcuberta, Phys. Rev. B 64, 180406 (2001)

310 [23] D. Niebieskikwiat, A. Caneiro, R.D. Sánchez, J. Fontcuberta, Phys. B 320, 107 (2002)

311 [24] L. Harnagea, B. Jurca, P. Berthet, J. Solid State Chem. 211, 219 (2014)

312 [25] D.D. Sarma, S. Ray, K. Tanaka, M. Kobayashi, A. Fujimori, P. Sanyal, H.R. Krishnamurthy, C. 313 Dasgupta, Phys. Rev. Lett. 98, 157205 (2007)

314 [26] E. Burzo, I. Balasz, S. Constantinescu, I.G. Deac, J. Magn. Magn. Mater. 316, e741 (2007)

315 [27] D. Niebieskikwiat, F. Prado, A. Caneiro, R.D. Sánchez, Phys. Rev. B 70, 132412 (2004)

316 [28] J.-F. Wang, B. Hu, J. Zhang, Z.-B. Gu, S.-T. Zhang, J. Alloys Compd. 621, 131 (2015)

317 [29] J.F. Wang, J. Zhang, B. Hu, Z.B. Gu, S.T. Zhang, J. Phys. D: Appl. Phys. 47, 445003 (2014)

318 [30] W. Zhong, W. Liu, C.T. Au, Y.W. Du, Nanotechnology 17, 250 (2006)

319 [31] M. Saloaro, S. Majumdar, H. Huhtinen, P. Paturi, J. Phys.: Condens. Matter 24, 366003 (2012)

320 [32] A. Nag, S. Jana, S. Middey, S. Ray, Indian J. Phys. 91, 883 (2017)

321 [33] F.J. Yue, S. Wang, L. Lin, H.F. Ding, D. Wu, Jo J. Phys. D: Appl. Phys. 45, 075001 (2012)

322 [34] S. Wang, F.J. Yue, D. Wu, F.M. Zhang, W. Zhong, Y.W. Du, Appl. Phys. Lett. 94, 012507 (2009)

323 [35] L. Xi, J.H. Du, J.H. Ma, Z. Wang, Y.L. Zuo, D.S. Xue, J. Alloys Compd. 550, 365 (2013)

324 [36] F.J. Yue, S. Wang, D. Wu, Appl. Phys. A 111, 347 (2013) 
Figures

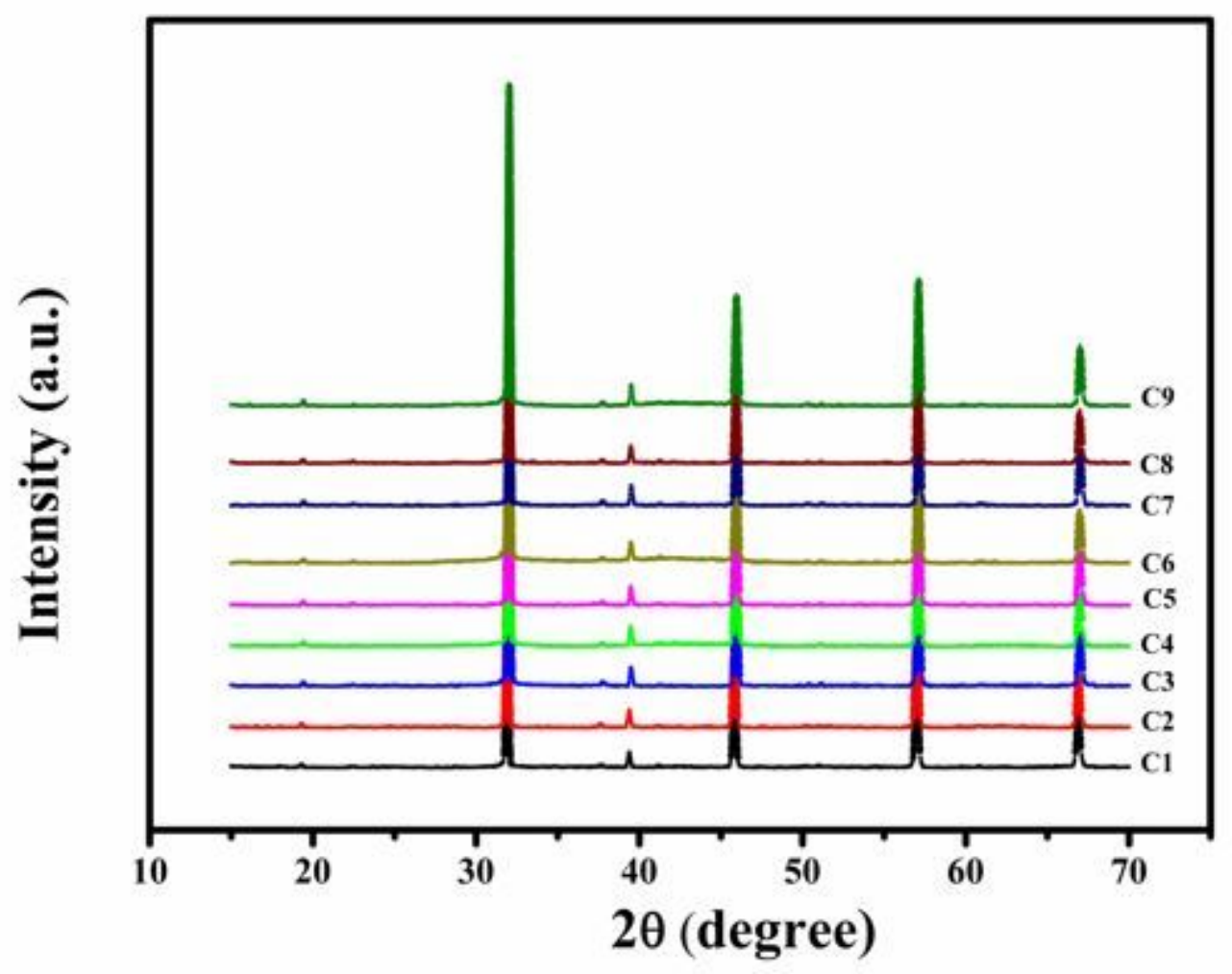

Figure 1

The XRD patterns of all prepared ceramics (C1-C9)
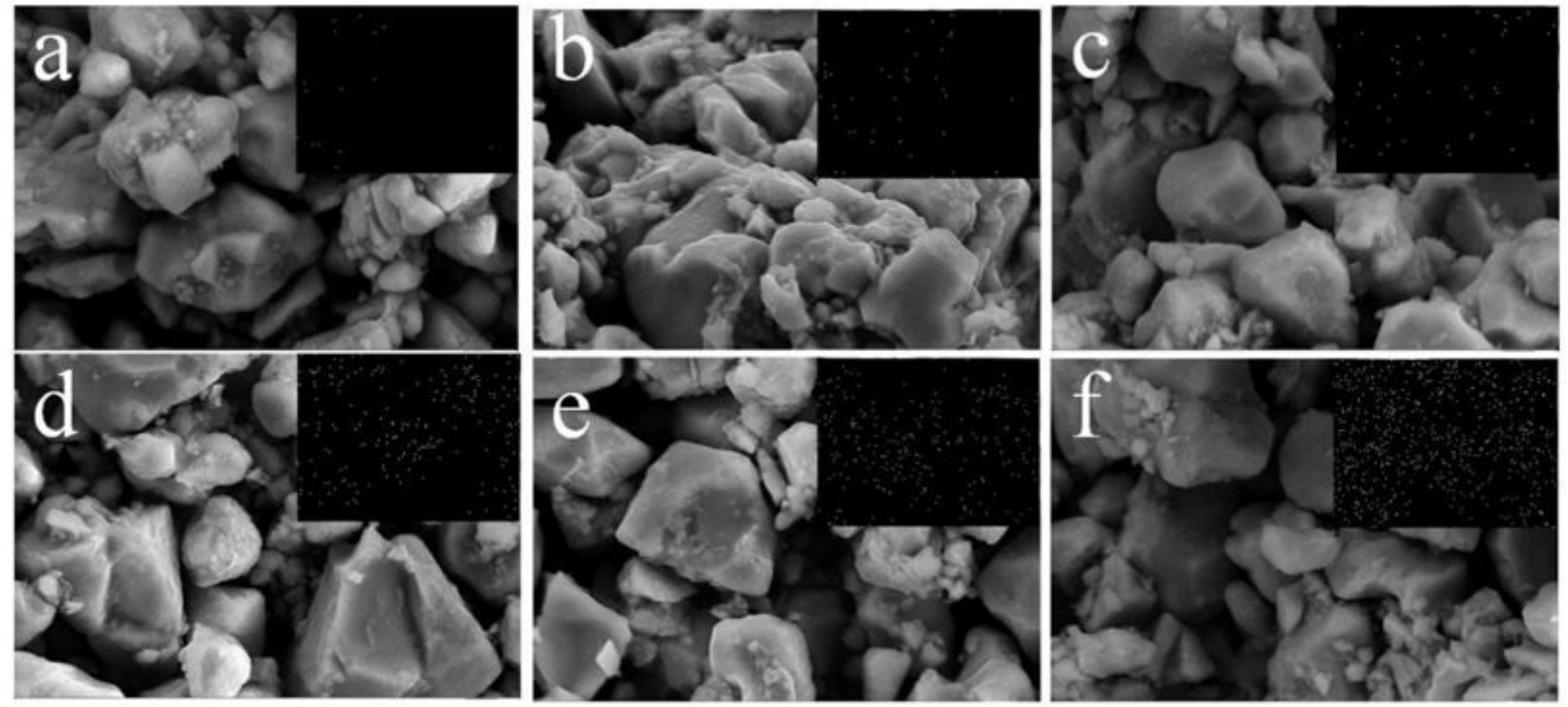
Figure 2

FESEM images of oleic acid/La0.5Sr1.5FeMoO6 composites, (a-f) correspond to C1-C6 samples. The insets are the corresponding $\mathrm{C}$ element mappings

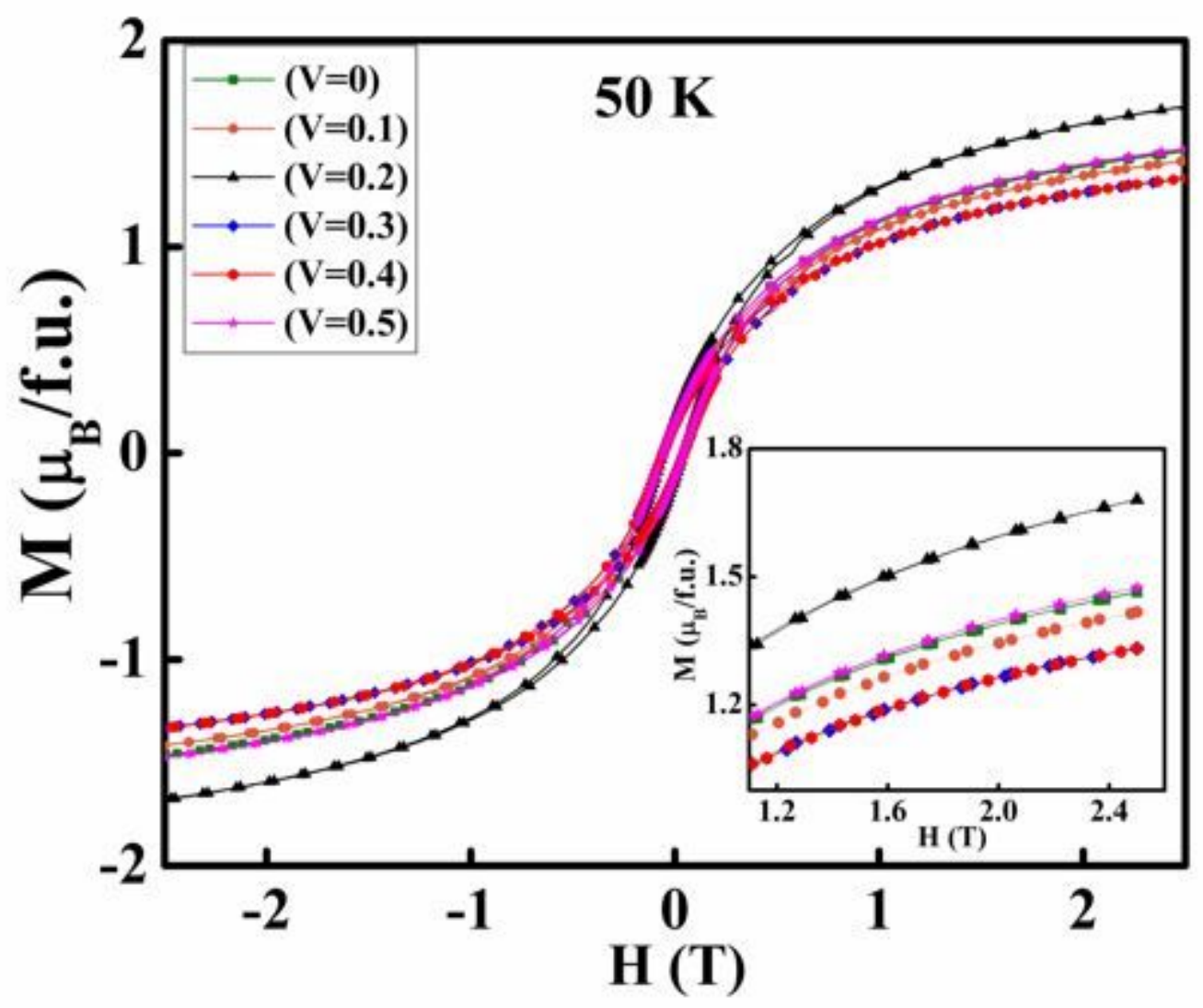

Figure 3

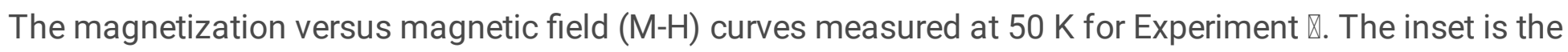
locally enlarged M-H curves 


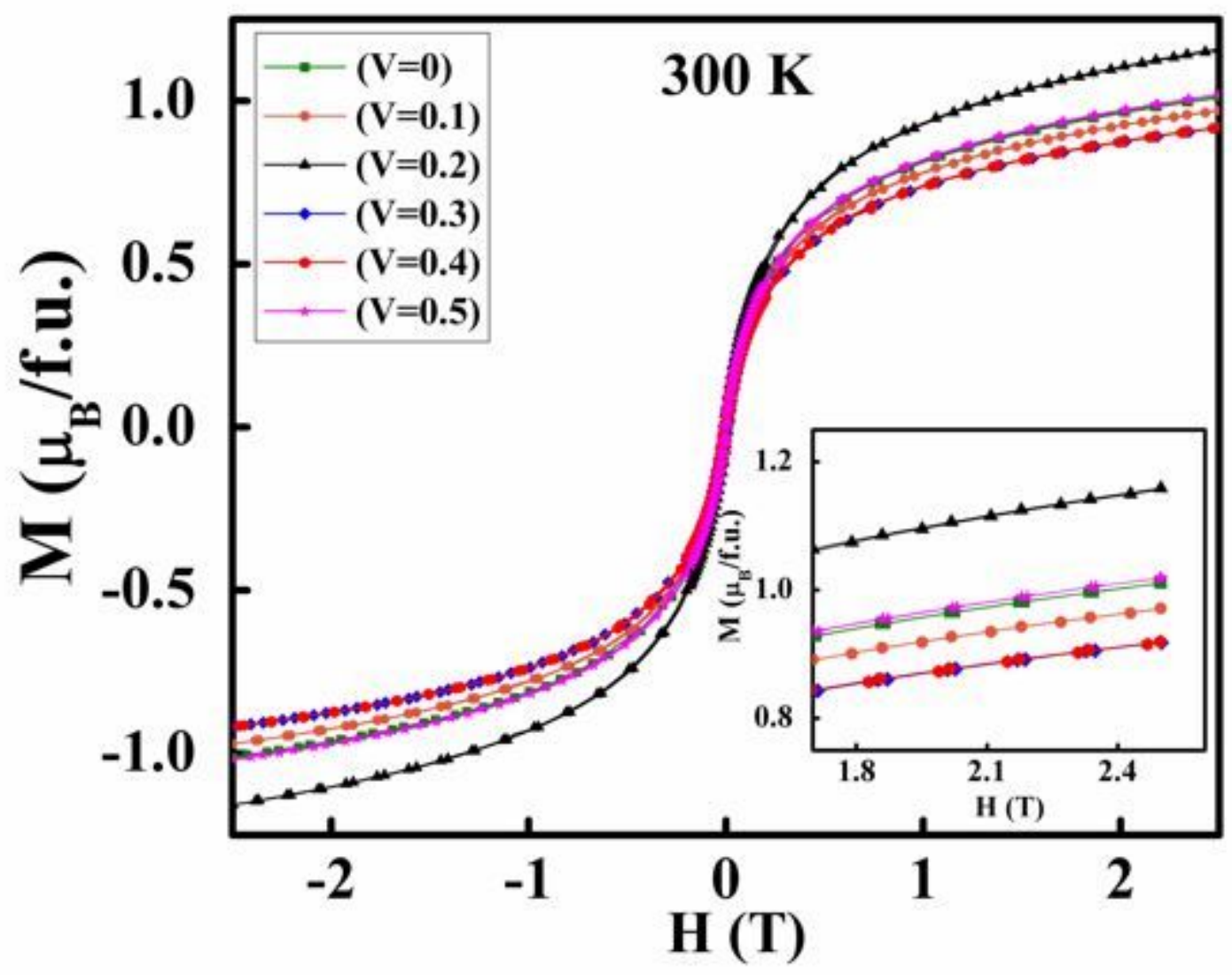

Figure 4

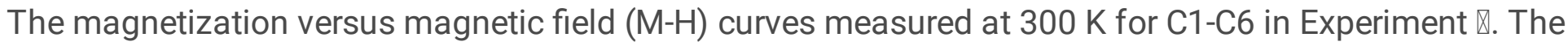
inset is the locally enlarged $\mathrm{M}-\mathrm{H}$ curves 

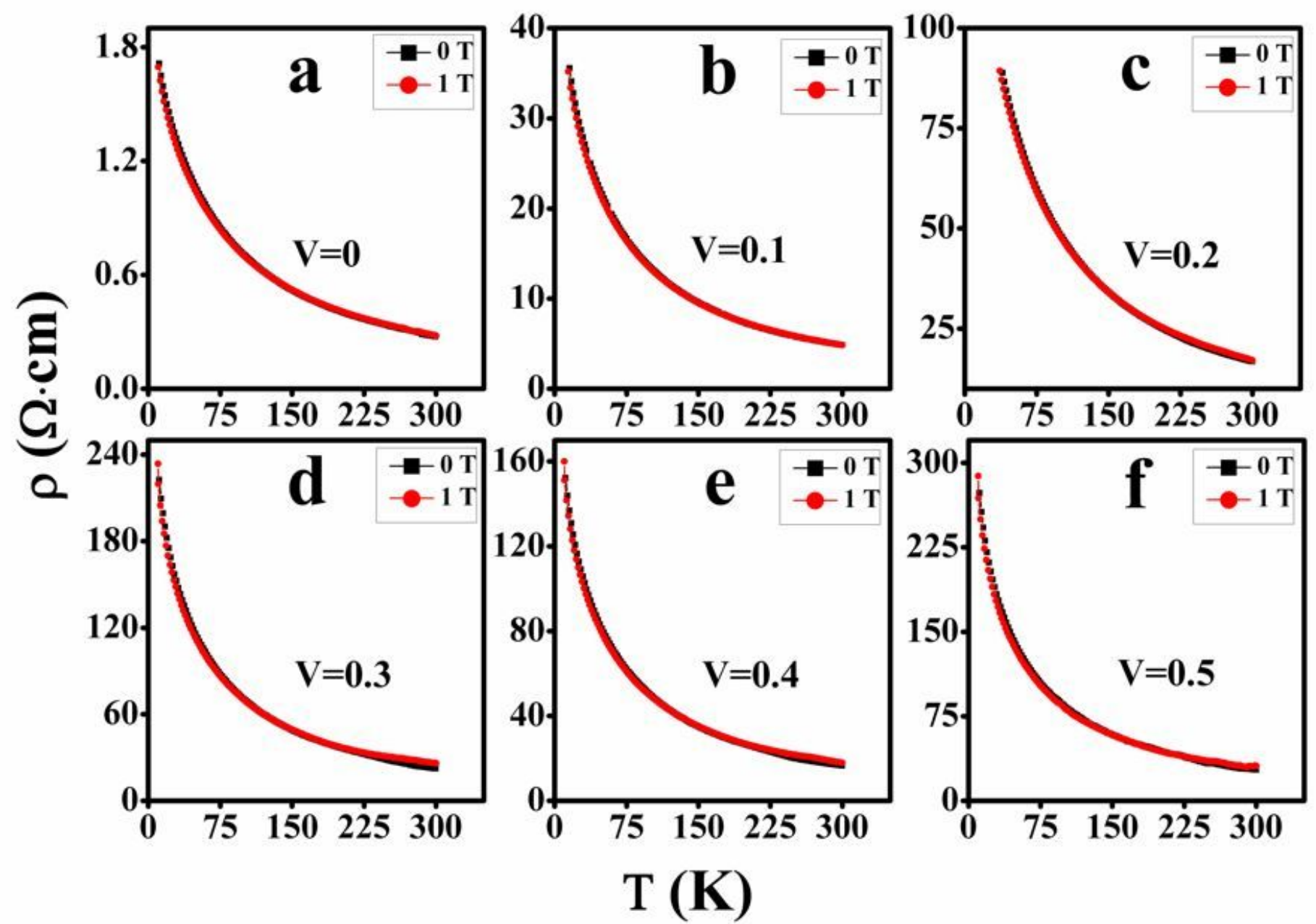

Figure 5

Temperature dependent resistivity curves for C1-C6 (a-f) measured at $10 \mathrm{~K}-300 \mathrm{~K}$ with $0 \mathrm{~T}$ and $1 \mathrm{~T}$ extra field 


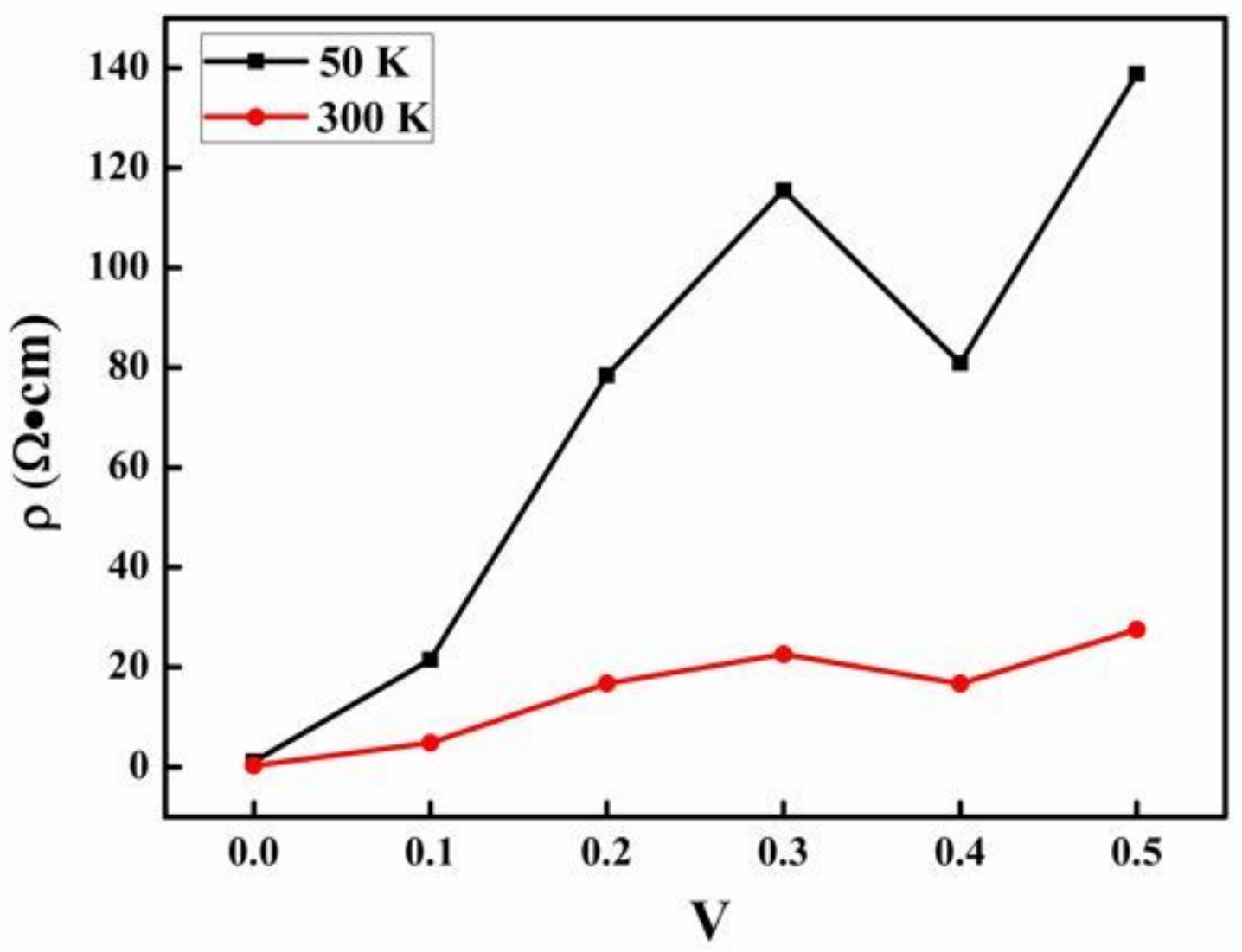

Figure 6

The resistivity-volume proportions curves of samples (C1-C6) at $50 \mathrm{~K}$ and $300 \mathrm{~K}$ with zero-field
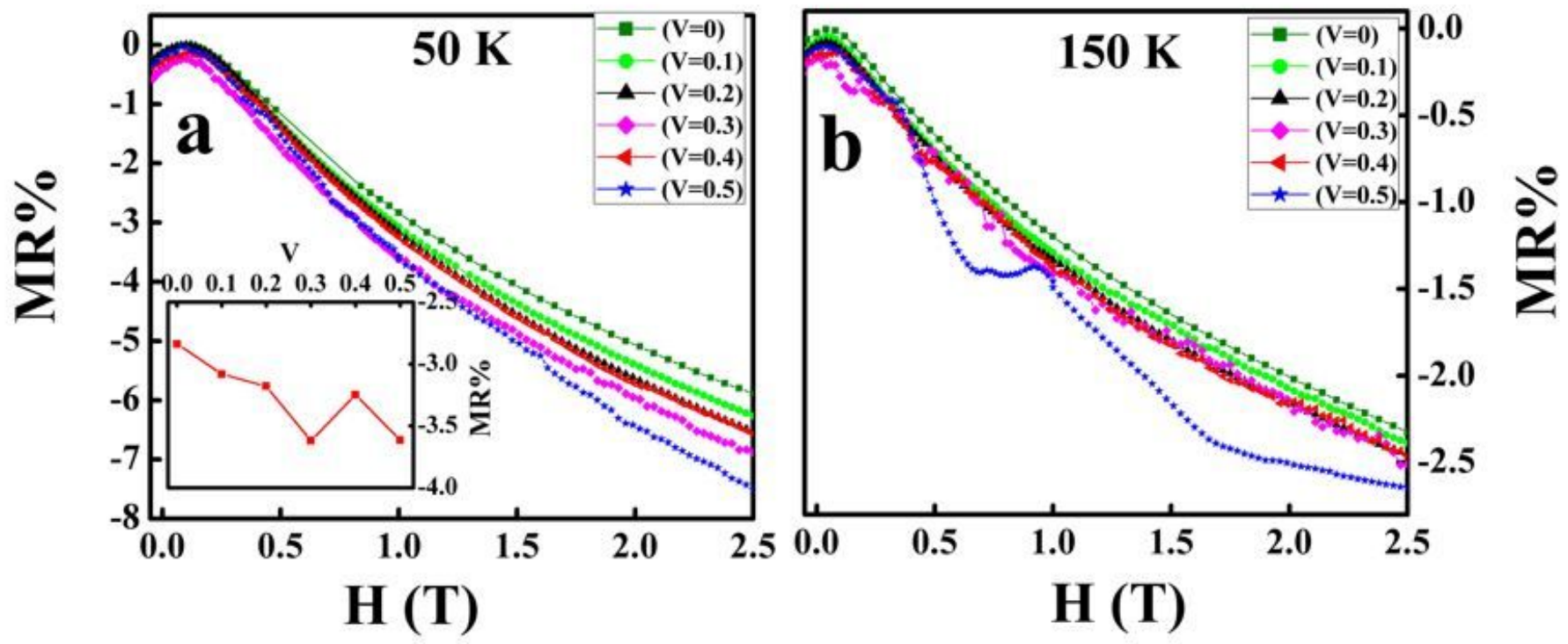
Figure 7

MR\% versus applied magnetic field plots of La0.5Sr1.5FeMoO6 with different volume proportions of oleic acid/alcohol contents $(\mathrm{V}=0,0.1,0.2,0.3,0.4,0.5)$ measured at $50 \mathrm{~K}(\mathrm{a})$ and $150 \mathrm{~K}(\mathrm{~b})$. The inset in (a) was the volume proportions dependent MR at $1 \mathrm{~T}$

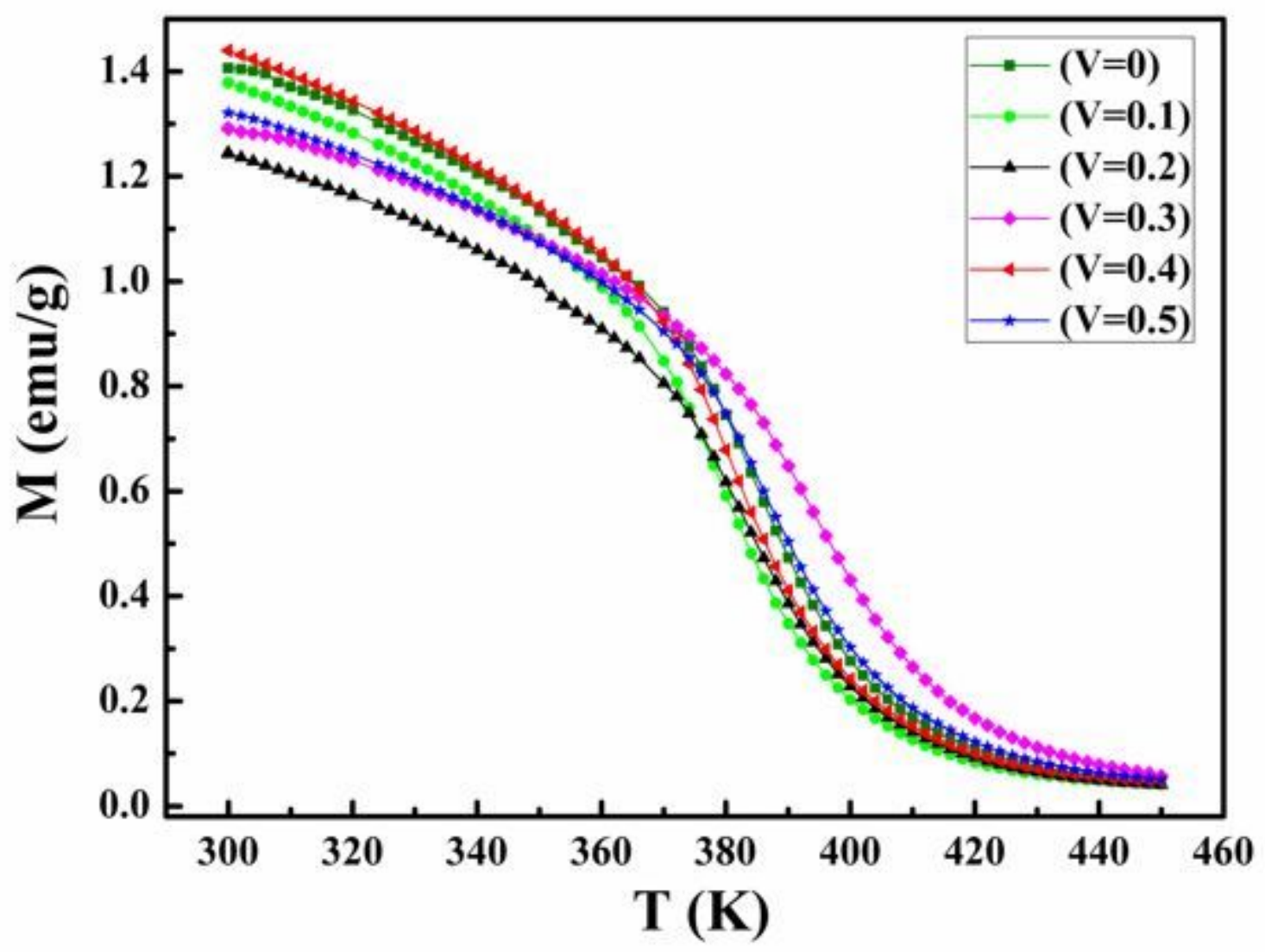

Figure 8

Magnetization-temperature (M-T) curves for C1-C6 measured from $300 \mathrm{~K}$ to $450 \mathrm{~K}$ 


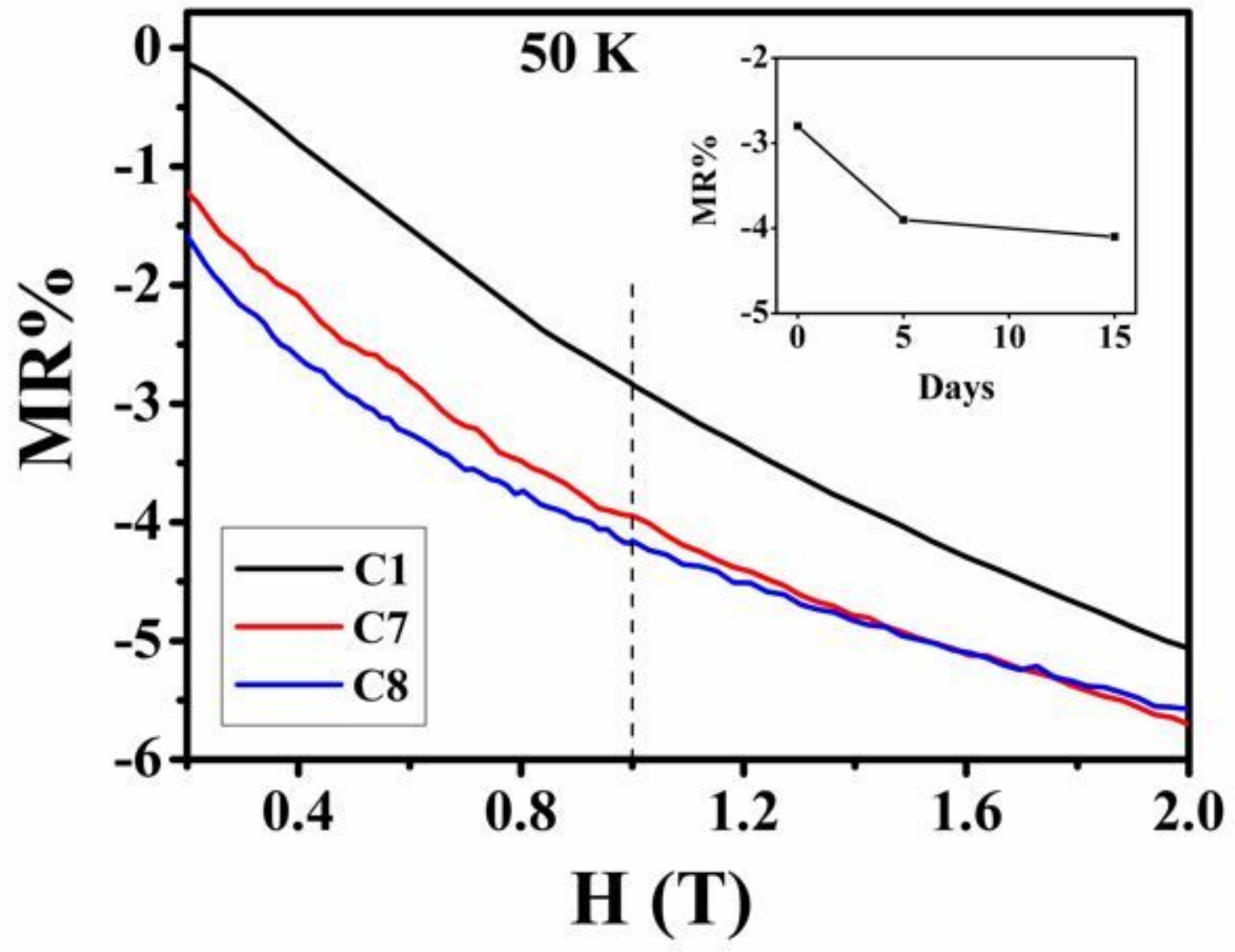

Figure 9

MR\% versus applied magnetic field of $\mathrm{C} 1, \mathrm{C} 7$ and $\mathrm{C} 8$ measured at $50 \mathrm{~K}$ (Experiment $\mathrm{Q}$ ). The inset is the LFMR value at $1 \mathrm{~T}$ of $\mathrm{C} 1, \mathrm{C} 7$ and $\mathrm{C} 8$ 


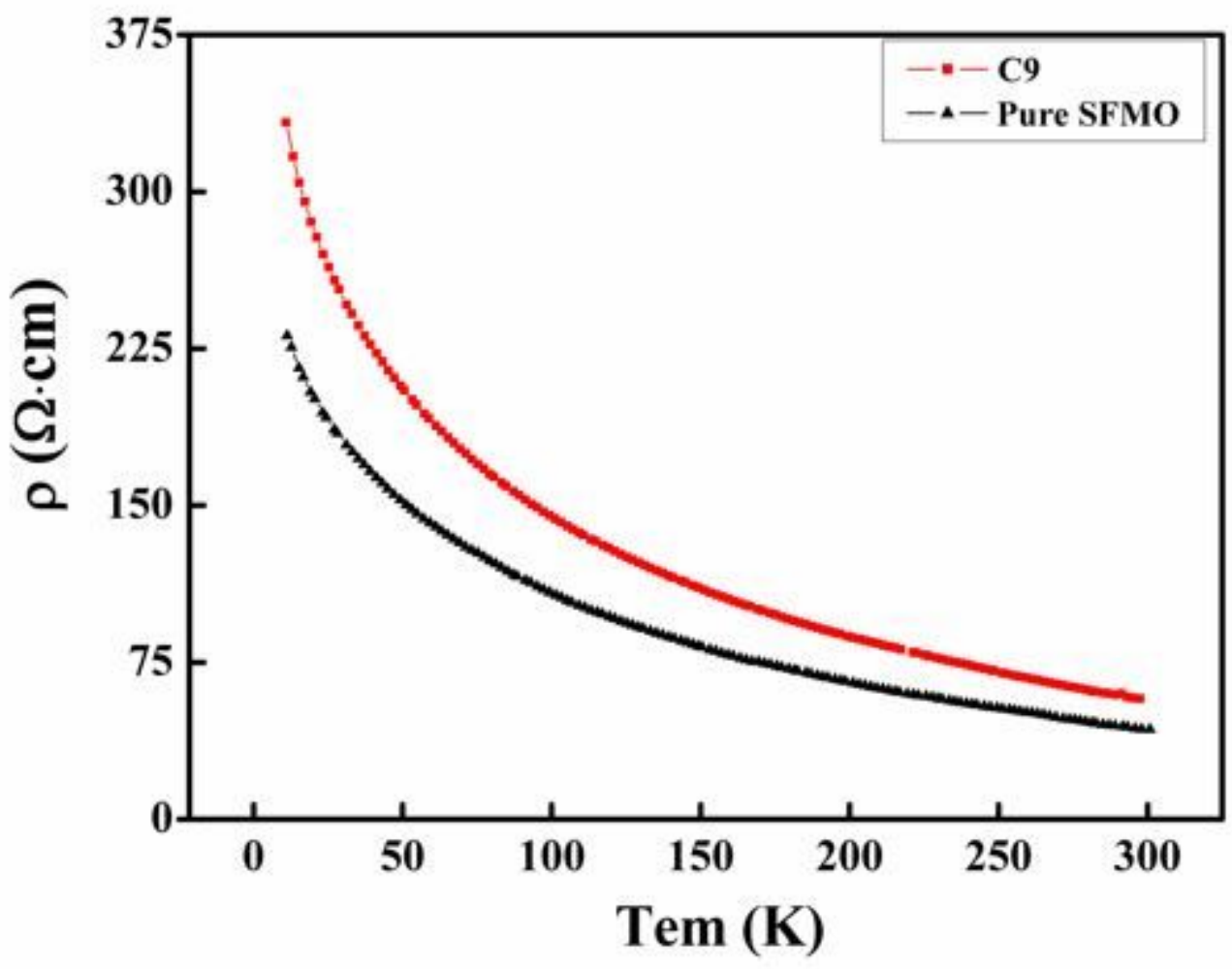

Figure 10

Resistivity versus temperature curves for pure SFMO and C9 at $10 \mathrm{~K}-300 \mathrm{~K}$ with zero-field 


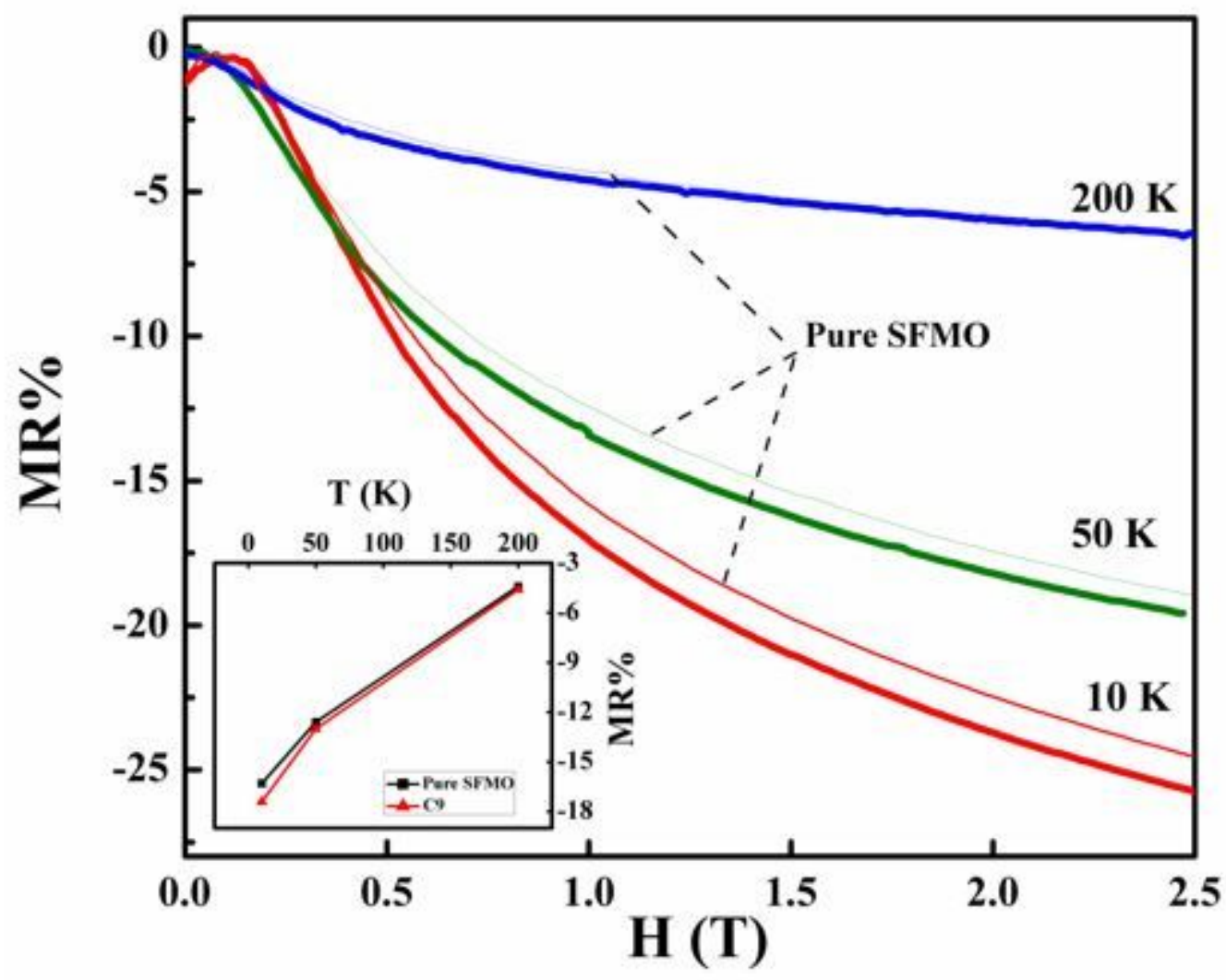

Figure 11

MR\% versus applied magnetic field of pure SFMO sample (thin lines) and C9 (thick lines) measured at 10 $\mathrm{K}, 50 \mathrm{~K}$ and $200 \mathrm{~K}$. The inset in figure is the measured temperature dependent low field magnetoresistance at $1 \mathrm{~T}$ for the two samples 